\title{
Cheating among college and university students: A North American perspective
}

\author{
Donald L. McCabe, \\ Rutgers University, USA \\ dmccabe@andromeda.rutgers.edu
}

\begin{abstract}
Academic integrity is an issue of critical importance to academic institutions and has been gaining increasing interest among scholars in the last few decades. This article discusses data obtained over the last three years from over 80,000 students and 12,000 faculty in the United States and Canada. While documenting that cheating on tests and exams and plagiarism are significant issues on our college and university campuses, it also offers some thoughts on possible strategies to encourage greater levels of academic integrity among students.
\end{abstract}

Keywords: academic integrity, cheating, honor codes

\section{Introduction}

It is a privilege to write an article for the inaugural issue of the International Journal for Educational Integrity. I have fond memories of making one of the keynote addresses at the first meeting of the Asia Pacific Educational Integrity Conference in Adelaide in 2003 and I am truly pleased to know that the release of this inaugural issue has been timed to coincide with the second APEI conference at the University of Newcastle.

The most appealing aspect of writing this article is the goal of this new journal to highlight some of the many issues related to questions of academic dishonesty that go beyond the difficult issue of plagiarism. While it is impossible to engage in a meaningful discussion of student cheating without at least some mention of plagiarism, it is my objective to focus on other forms of academic dishonesty as well and to discuss how students perceive academic dishonesty in a more global sense. While I have extensive data to support such a discussion, most of it has come from students in the United States (US) and Canada. Although there are a few notable differences, the similarities in the data collected in the US and Canada suggest the basic lessons to be learned are relevant to many countries in the Asia Pacific region, especially Australia and New Zealand. However, this assumption lacks a strong empirical basis.

\section{My Surveys}

Although I have been surveying students and faculty since 1990, the data discussed here were collected during the last three academic years using a web-based survey on 83 different campuses in the US (67 campuses) and Canada (16 campuses). While undergraduate students have provided most of this information, I will also discuss the data provided by graduate students and faculty. Readers interested in earlier phases of this ongoing project might be interested in one of the following articles: McCabe and Trevino (1996) or McCabe, Trevino and Butterfield (2001, 2004).

Most of this data has been generated as part of the Academic Integrity Assessment Project conducted by the Center for Academic Integrity at Duke University in the US. Schools participating in this project are trying to get the 'pulse' of academic integrity on their campuses. In addition to assessing student and faculty perceptions of the overall climate of academic integrity on a campus, the survey asks students to self-report instances of academic dishonesty in which they have personally engaged. While self-report data is one of the 
few ways to obtain a general picture of the cheating climate or culture on a campus, it raises significant issues of possible response bias in the resulting data and, because of its potentially sensitive nature for some students, many elect not to participate in the survey.

Typically, participating campuses send an email invitation to all undergraduate and graduate students inviting them to complete a survey. Although it is almost impossible to generate accurate response rates since there is no way of confirming how many students actually receive an invitation, it is clear the response rate is below desired levels, averaging between $10 \%$ and $15 \%$ and varying from as little as $5 \%$ to $10 \%$ on some large campuses to over $50 \%$ on a limited number of small, residential campuses. This contrasts with return rates in the range of $25-30 \%$ for written surveys I have conducted. While I am confident that the data still provide a reasonable relative indication of the level of academic dishonesty on different campuses, these low return rates and potential response bias should be kept in mind when interpreting any survey results.

The good news associated with using web surveys is the ease with which one can accommodate larger samples. And, as shown in Table 1, a large number of both students and faculty have participated in my survey process over the last three years.

Table 1

Survey Participants - 2002 to 2005

\begin{tabular}{|c|c|c|c|}
\hline & United States & Canada & Total \\
\hline \multicolumn{4}{|l|}{ Students } \\
\hline Undergraduate & 51,611 & 19,460 & 71,071 \\
\hline Graduate & 9,080 & 2,199 & 11,279 \\
\hline Faculty & 9,740 & 2,576 & 12,316 \\
\hline Teaching Assistants & 710 & 1,037 & 1,747 \\
\hline
\end{tabular}

While response rates and response bias are of concern, clearly this is still a very rich database.

\section{Academic Dishonesty \\ Tests and Examinations}

In general, my survey has included six behaviors related to tests and examinations: copying from another student on a test or exam with and without his/her knowledge; using unauthorised crib notes or cheat notes during a test or exam; learning what will be on a test from someone who has taken it in an earlier period; helping someone else cheat on a test or exam; and the use of a false or forged excuse to delay taking an test/exam or submitting a written assignment, thus gaining more time to study or prepare an assignment and/or being able to find out from someone who has taken a test what was on it, how difficult it was, or what areas to focus on in their test preparation. (Although this behavior asked about delaying both tests/exams and the submission of written work, it is discussed here since open-ended comments provided by students suggest it may be a more common strategy with regard to delaying the taking of a test. But clearly some students use false excuses to extend the due date on written assignments.) More recently, I have also added a question that asks students about the inappropriate use of electronic devices (e.g., Palm Pilots and cell phones) to obtain information during a test or exam. In addition to asking students whether they have engaged in any of these behaviors in the past year, I also ask them to assess how serious they consider each behavior to be - not cheating, trivial cheating, moderate cheating or serious cheating. I have addressed the same questions to faculty, first asking them to think about the number of times they have actually observed any of these behaviors during the last three years. And, as I did with students, I also ask faculty to assess the seriousness of each of these behaviors. While some differences are observed in student behaviors between the United States and Canada, among students at different types of schools (e.g., two year community colleges in the US, four year liberal arts colleges and universities) and across such factors as choice of major, gender, age, 
etc., for ease of presentation I will focus on aggregate findings and highlight the differences that seem most important or interesting.

Table 2 presents the aggregate information for the frequency of different test and exam behaviors (student self-reports of their own engagement in each behavior in the past year and faculty reports of the different behaviors they have observed in one or more of their courses in the past three years).

Table 2

Cheating on Tests and Examinations*

$\underline{\text { Undergraduates }}$ Grad Students $\quad \underline{\text { Faculty }}$

Learning what is on a test from someone who has already taken it

$33 \%$

$17 \%$

$35 \%$

Using false excuse to delay taking test

$16 \%$

$9 \%$

$49 \%$

Copying from another student on a test/exam without their knowledge

$11 \%$

$4 \%$

$41 \%$

Helping someone else cheat on test

$10 \%$

$6 \%$

$29 \%$

Copying from another student on a

test/exam with their knowledge

$9 \%$

$3 \%$

$33 \%$

Using unauthorised crib/cheat notes

$8 \%$

$4 \%$

$26 \%$

Using an electronic/digital device as an

unauthorised aid during a test/exam

$5 \%$

$2 \%$

$11 \%$

Note: Values represent \% of students who have engaged in the behavior at least once in the past year or faculty who have observed the behavior in a course in the last three years.

*N exceeds 64,000 responses for undergraduates, 9,700 for graduate students and 9,650 for faculty in all cases except using an electronic/digital device. This question has only appeared on the most recent version of my survey and $\mathrm{N}=18,177$ for undergraduates, 4,618 for graduate students and 2,932 for faculty.

Table 2 leads to several important conclusions. First, cheating on tests and exams among undergraduate students is problematic on US and Canadian campuses, as roughly one in ten students admit to one or more instances of copying, using crib notes and/or helping someone else to cheat on a test or exam. Indeed, if the number of students who admit to any of these four offenses in the past year is calculated, it is evident that one in five students (21\%) has engaged in at least one of these serious forms of test or exam cheating. Second, somewhat surprisingly in light of recent media stories to the contrary, the data in Table 2 suggest that the use of electronic devices during tests and exams is modest. Although a result that suggests one in twenty students admits to such behavior is still troubling, it is far below the levels suggested by many in the media. However, in light of the issues of response bias and low return rates discussed earlier, the $5 \%$ figure probably understates the actual problem and, since it is still a relatively new phenomenon, it is very possible there will be some further growth in this behavior in the future. Third, one in seven students (16\%) acknowledges s/he has used a false excuse to delay taking an exam (or submitting a written assignment), sometimes with the goal of simply getting more time to study or work but at least occasionally with the objective of learning what was on the test before s/he has to take it. Even if the test or exam is not identical, students seem to feel knowing the format of a test and basic areas to emphasize in their preparation is helpful. Indeed, a third of 
students (33\%) have obtained such 'pretest' information one or more times in the past year, although typically by simply speaking to a peer who has taken the test in an earlier class period rather than actually delaying the taking of their own exam. Fourth, while self-reported rates are typically half or less of reported undergraduate rates, it is evident that graduate students are certainly not immune to cheating. One in ten admits to one of the four more serious forms of cheating, $2 \%$ acknowledge using an electronic advice during a test or exam, 9\% have used a false excuse to delay taking an exam or submitting a written assignment and $17 \%$ have obtained information about a test from a peer who has taken it in an earlier period. Finally, as might be expected, faculty report even higher levels of observed cheating, with one quarter to one half of faculty respondents reporting an observation in every category of test/exam cheating, with the exception of using an electronic device as an aid during a test or exam, which only $11 \%$ of faculty say they have observed.

Although the primary focus in this study is on aggregate results, as mentioned earlier, there are some notable differences in selected results for different subgroups. For example, confirming findings of earlier phases of this project (e.g., McCabe \& Trevino, 1995), it has been found that undergraduates majoring in business selfreport among the highest levels of the more serious forms of test and exam cheating (copying from another, using crib notes and helping another to cheat) - a mean self-reported rate of $26 \%$ for undergraduate business students versus a mean of $20 \%$ for all other undergraduates. In addition, male undergraduates self-report higher levels of more serious forms of test/exam cheating than females $-24 \%$ versus $20 \%$. Finally, there are some country differences between US and Canadian students on serious test and exam cheating. In particular, significantly more US faculty (54\%) versus Canadian faculty (45\%) report observing such cheating, a result that is supported by the greater number of US students (22\%) versus Canadian students (19\%) that self-report serious test/exam cheating. The same trend can be seen among graduate students, but the differences in this case are fairly trivial - $10 \%$ for US graduate students versus $9 \%$ for Canadian graduate students.

Student and faculty perceptions of the seriousness of the different forms of test and examination cheating are summarized in Table 3, where percentage of students and faculty who rate the specified behaviors as either moderate or serious cheating (versus the choices of not cheating or trivial cheating) have been calculated.

Table 3

Perceived Seriousness of Different Behaviors Related to Test and Examinations

$\underline{\text { Undergraduates }}$ Grad Students $\quad \underline{\text { Faculty }}$

Learning what is on a test from someone who has already taken it

$\begin{array}{rrr}64 \% & 79 \% & 93 \% \\ 58 \% & 67 \% & 80 \% \\ 92 \% & 95 \% & 98 \% \\ 89 \% & 93 \% & 98 \% \\ 91 \% & 95 \% & 99 \% \\ 90 \% & 93 \% & 98 \% \\ 90 \% & 93 \% & 98 \%\end{array}$

Using false excuse to delay taking test

Copying from another student on a test/exam without their knowledge

Helping someone else cheat on test

Copying from another student on a test/exam with their knowledge

Using unauthorised crib/cheat notes

Using unauthorized electronic device to obtain information during test/exam

$90 \%$ $98 \%$

Note: Values represent \% of students and faculty who rate the behavior as moderate or serious cheating versus choices of not cheating or trivial cheating. 
The first obvious conclusion suggested by Table 3 is that the vast majority of students realize that copying from another student on a test or exam, using unauthorised crib or cheat notes, helping someone else to cheat on a test or exam and using an unauthorized electronic device to obtain information during a test or exam constitute moderate or serious cheating. Indeed, slightly over $90 \%$ of undergraduates and $94 \%$ of graduate students on average so rate each of these five behaviors although the previous data indicated that more than one in five students self-report having engaged in one or more of these behaviors.

Such data may facilitate understanding of the potential difficulties of eliminating or significantly reducing serious test cheating. Using unauthorised crib or cheat notes as a typical example, the undergraduate student sample can be divided into two groups - those who acknowledge they have engaged in this behavior and those who have not. How each of these groups rates the seriousness of using crib or cheat notes during a test or exam can then be examined. As might be expected, a much higher percentage of the undergraduates who have not engaged in this behavior (92\%) rate it as moderate or serious cheating versus the lower rating (72\%) provided by those who acknowledge using crib notes. This seems to suggest two distinct challenges to any efforts to reduce the inappropriate use of crib or cheat notes, or other forms of academic dishonesty. First, there certainly is a need to develop strategies to educate students who use crib notes (or engage in other serious forms of test/exam cheating) and do not believe this is an inappropriate behavior. This will not be easy but perhaps the even greater challenge will be addressing what might be described as the more hardened cheaters, those who use crib notes even though they seem to realize it is moderate or serious cheating. Based on the many comments students have provided on their surveys, there are a number of factors involved in such judgments, including a variety of situational factors. For example, student comments suggest that cheating is greater, and they believe more justifiable, in courses where the faculty member tends to ignore obvious incidents of cheating that occur (putting honest students at a disadvantage), where faculty recycle exams and written assignments, in courses which students are required to take to fulfill a distribution requirement but which, at least in the minds of students, have little relation or value to their intended major. These issues will be returned to later.

\section{Written Assignments}

As worrisome as cheating on tests and examination may be, cheating on written work seems to occur even more frequently, as suggested in Table 4.

It is clear from Table 4, that unauthorised collaboration, paraphrasing or copying a few phrases or sentences from either a written or web source ('cut and paste' plagiarism) and fabricating or falsifying a bibliography occur frequently, being reported by one quarter to one half of undergraduates and as many as one quarter of graduate students. In addition, over two-thirds of faculty report they have observed cut and paste plagiarism. At the other end of the spectrum - the four behaviors involving submitting work that is not your own - there are much lower levels of engagement, but still not trivial. For example, if the four behaviors in which students engage least frequently (turning in work copied from another, copying large sections of text from written sources, turning in work done by another and downloading or otherwise obtaining a paper from a term paper mill or website) are combined, it is clear that $16 \%$ of all undergraduate respondents and $8 \%$ of responding graduate students report one or more of these behaviors in the past year. In contrast, a surprisingly large number of faculty (79\%) report they have observed one or more instances of these behaviors in the last three years, driven in part by a perception that a large number of students (59\%) have copied material almost word for word from a written source without citation. While this behavior is intended to capture larger scale plagiarism, it is possible that the perceived high level of student engagement reported by faculty is driven by instances which students view as less egregious and place in the 'cut and paste' category. 
Table 4

Cheating on Written Assignments*

\section{Undergraduates Grad Students $\underline{\text { Faculty }}$}

Working with others on an assignment

when asked for individual work

$42 \%$

$26 \%$

$60 \%$

Paraphrasing/copying few sentences from

written source without footnoting it

$38 \%$

$25 \%$

$80 \%$

Paraphrasing/copying few sentences from

Internet source without footnoting it

$36 \%$

$24 \%$

$69 \%$

Receiving unpermitted help from

someone on an assignment

$24 \%$

$13 \%$

$44 \%$

Fabricating/falsifying a bibliography

$14 \%$

$7 \%$

$34 \%$

Turning in work copied from another

$8 \%$

$4 \%$

$38 \%$

Copying material almost word for word

from a written source without citation

$7 \%$

$4 \%$

$59 \%$

Turning in work done by another

$7 \%$

$3 \%$

$45 \%$

Obtaining paper from term paper mill

$3 \%$

$2 \%$

$29 \%$

Note: Values represent \% of students who have engaged in the behavior at least once in the past year or faculty who have observed the behavior in a course in the last three years.

*N exceeds 63,700 responses for undergraduates, 9,250 for graduate students and 9,000 for faculty in all cases.

But the most interesting result in Table 4 may be the fact that undergraduates, graduate students and faculty all report engaging in or observing slightly lower levels of internet plagiarism versus plagiarism from written sources. Although this finding runs counter to recent media reports, it is a result I have observed consistently in my surveys over the last four or five years. A partial explanation may be that there is some confusion in the minds of students, and faculty, as to exactly what each question is seeking. For example, if a student engages in 'cut and paste' plagiarism from a journal article via the web does s/he consider that to be plagiarism from a written source (the original format of the source) or Internet plagiarism (the means of access)? Another factor may be that while the web may offer a greater variety of easily accessed sources, an increasing number of students suggest that, depending on the course and assignment, they are turning to written sources which they believe may not be as easily detected through a Google search or plagiarism detection software as something that comes directly from the web. Supporting these arguments to at least some degree is the observation that almost two-thirds (62\%) of undergraduates and 59\% of graduate students who report engaging in 'cut and paste' plagiarism at all, have engaged in such plagiarism from both written and Internet sources. Twenty percent of undergraduates (and 22\% of graduate students) who acknowledge 'cut and paste' plagiarism, report using written sources only and $18 \%$ of both undergraduate and graduate students indicate they rely solely on the web. 
Table 5

Perceived Seriousness of Different Behaviors Related to Written Work

\begin{tabular}{|c|c|c|c|}
\hline & Undergraduates & Grad Students & Faculty \\
\hline $\begin{array}{l}\text { Working with others on an assignment } \\
\text { when asked for individual work }\end{array}$ & $32 \%$ & $54 \%$ & $82 \%$ \\
\hline $\begin{array}{l}\text { Paraphrasing/copying few sentences from } \\
\text { written source without footnoting it }\end{array}$ & $56 \%$ & $68 \%$ & $84 \%$ \\
\hline $\begin{array}{l}\text { Paraphrasing/copying few sentences from } \\
\text { Internet source without footnoting it }\end{array}$ & $57 \%$ & $68 \%$ & $82 \%$ \\
\hline $\begin{array}{l}\text { Receiving unpermitted help from } \\
\text { someone on an assignment }\end{array}$ & $44 \%$ & $65 \%$ & $85 \%$ \\
\hline Fabricating/falsifying a bibliography & $58 \%$ & $74 \%$ & $90 \%$ \\
\hline Turning in work copied from another & $88 \%$ & $92 \%$ & $98 \%$ \\
\hline $\begin{array}{l}\text { Copying material almost word for word } \\
\text { from a written source without citation }\end{array}$ & $91 \%$ & $94 \%$ & $99 \%$ \\
\hline Turning in work done by another & $86 \%$ & $93 \%$ & $98 \%$ \\
\hline Obtaining paper from term paper mill & $89 \%$ & $92 \%$ & $98 \%$ \\
\hline
\end{tabular}

Note: Values represent percent of students and faculty who rate behavior as moderate or serious cheating versus choices of not cheating or trivial cheating.

As occurred with test and exam cheating, one of the most obvious conclusions from Table 5 is that the vast majority of students realize that significant levels of plagiarism and turning in written work either copied from or done by another student constitute moderate or serious cheating. Almost $90 \%$ of undergraduates and $92 \%$ or more of graduate students so rate each of these behaviors, as do $98 \%$ or more of faculty. Yet, as in the case of test and exam cheating, this does not stop students from at least occasionally engaging in these behaviors. As noted above, $16 \%$ of undergraduates and $8 \%$ of graduate students self-report having engaged in one or more of these behaviors and, for the reasons suggested earlier, it can probably be assumed that the actual level is higher than this.

But unlike cheating on tests and exams, in the case of written cheating there are two behaviors (unpermitted collaboration or receiving unpermitted help on an assignment) which only a minority of students view as moderate or serious cheating. Unpermitted collaboration is a particularly difficult issue as barely a majority (53\%) of even those undergraduates who apparently do not engage in such behavior do not do so because they consider it moderate or serious cheating. With growing pressure from prospective employers on universities to help students learn how to work together collaboratively, any faculty member who thinks his/her simple admonitions to the contrary will prevent such collaboration is probably being naïve. In addition, as an increasing number of students hold jobs to pay for their education and/or try to balance extracurricular activities with the demands of their academic work, it becomes very easy for students to justify collaborative efforts, especially on assignments they conclude have limited learning value or place demands on students which they consider excessive. 
Also troubling in Table 5 is the fact that over $40 \%$ of undergraduates and $30 \%$ of graduate students (and almost $20 \%$ of faculty) are apparently not convinced that 'cut and paste' plagiarism is moderate or serious cheating. Although it is not known whether the levels of 'cut and paste' plagiarism are high because students don't consider it cheating, or whether the number of students who perceive 'cut and paste' plagiarism as cheating is so low because students are rationalizing their behavior, both scenarios are troubling and difficult to address. However, before turning to possible strategies to address student cheating, it is important to first consider cheating on other assignments.

\section{Other Assignments}

Although the data summarized in Table 6 suggest lower to moderate levels of cheating on other assignments, student comments suggest this may be misleading. Even though students were provided the response option of 'Not relevant' for each of the behaviors on the survey, the comments of many suggest they have answered the questions on laboratory, computer and research data in the negative even when they have had no or very limited opportunities to engage in these behaviors. Once again, even though these data are probably understated, they are still troublesome. For example, one in five undergraduates (19\%) suggests s/he has falsified laboratory data and approximately one in ten indicate they have copied someone else's computer program or code (11\%) or fabricated or falsified research data (8\%). And if undergraduates majoring in science are isolated, 31\% report falsifying laboratory data. However, there is not a dramatically higher level of students who acknowledge they have copied someone else's computer program among computer science majors - $15 \%$ versus $11 \%$.

Table 6

Cheating on Other Assignments*

$$
\text { Undergraduates } \quad \text { Grad Students } \quad \text { Faculty }
$$

Fabricating or falsifying lab data

$19 \%$

$7 \%$

$21 \%$

Copying someone else's program in a course requiring computer work

$11 \%$

$8 \%$
$7 \%$

$4 \%$
$39 \%$

$21 \%$

Note: Values represent \% of students who have engaged in the behavior at least once in the past year or faculty who have observed the behavior in a course in the last three years.

*N exceeds 46,000 responses for undergraduates, 7,000 for graduate students and 4,250 for faculty in all cases.

The explanation for these fairly modest levels of self-reported cheating may be found in Table 7 which suggests that, with the exception of undergraduate student ratings of fabricating or falsifying laboratory data, a significant majority of students perceive these other behaviors to be moderate or serious cheating. However, student comments suggest an even greater number of students would agree that fabricating or falsifying laboratory data is moderate or serious cheating but that they are able, at least occasionally, to justify such behavior on the basis of the poor equipment and facilities they are expected to use in their laboratorys. Students talk about broken or damaged scales which are known to give incorrect weights in chemistry experiments, laboratory instructors who refuse to replace needed materials that may have been damaged or destroyed in the course of an experiment, and the need to share laboratory facilities with other students, thus significantly limiting the amount of time a student has to complete his/her work. While it could be argued that none of these problems justifies cheating, such problems do make it easier to understand the behavior of some students. 
Table 7

Perceived Seriousness of Different Behaviors Related to Other Assignments

\begin{tabular}{|c|c|c|c|}
\hline & $\underline{\text { Undergraduates }}$ & $\underline{\text { Grad Students }}$ & Faculty \\
\hline Fabricating or falsifying lab data & $68 \%$ & $86 \%$ & $97 \%$ \\
\hline $\begin{array}{l}\text { Copying someone else's program in } \\
\text { a course requiring computer work }\end{array}$ & $83 \%$ & $89 \%$ & $97 \%$ \\
\hline Fabricating or falsifying research data & $80 \%$ & $91 \%$ & $98 \%$ \\
\hline
\end{tabular}

Note: Values represent \% of students and faculty who rate the behavior as moderate or serious cheating versus choices of not cheating or trivial cheating.

\begin{abstract}
What Next?
While these data may be interesting and informative, the major objective of my work over the last fifteen years has been to help colleges and universities think about strategies to improve the climate of academic integrity on their campuses. Although there clearly is a place for prevention and detection in any such strategies, the primary emphasis of my work has been on ways in which campuses can promote integrity among students. For me, reducing the level of cheating among students, while desirable, is not enough. Rather, I am more interested in helping students learn to accept responsibility not only for their own behavior, but also the well-being of the entire campus community. Many of the recommendations my colleagues and I have offered in this regard have already been discussed in the literature and will only be briefly reviewed here. Readers interested in greater detail may wish to consult one or more of the sources cited below. For ease of discussion, I will organize these comments into two parts - strategies individual faculty members might employ in their courses and institutional strategies, including the concept of honor codes.
\end{abstract}

\title{
Faculty Strategies to Promote Student Academic Integrity
}

It is clear that many faculty occasionally ignore incidents of suspected academic dishonesty in their courses. In the surveys discussed here, $41 \%$ of faculty acknowledge having done so and the primary reason they offer is the burden of proof required to prove a student has cheated. They frequently complain of cases where 'obviously guilty' students have been found not responsible. For many this is enough to convince them that it is not worth pursuing suspected cases in the future unless they truly have a smoking gun. If they have not had such an experience personally, they know a colleague who has. Unfortunately, such inaction in the face of cheating leads to even higher levels of cheating as students quickly become aware of which faculty are not likely to pursue cases of suspected cheating and their courses become targets for cheaters. This is why one of the most fundamental recommendations my colleague Gary Pavela and I have suggested in developing a set of principles of academic integrity for faculty is the need to respond to incidents of academic dishonesty when they occur. As we suggest in our principles, "Faculty members who ignore or trivialize academic dishonesty send the message that the core values of academic life aren't worth enforcing” (McCabe \& Pavela, 2004, p. 15). This not only emboldens students who are willing to cheat, but it creates a significant dilemma for other students. In my surveys, one of the most common rationales that students offer for cheating is the question of fairness. Students who claim they normally do not cheat feel they have no choice when a faculty member makes little or no effort to prevent or respond to cheating. In the highly competitive market for top grades, many students have convinced themselves that cheating is their only choice when faculty 'let' other students cheat. In fact, several of the faculty principles that Pavela and I have suggested focus on reducing opportunities for students to cheat, making expectations on tests and assignments clear for students and using creative forms of assessment that challenge students and help to truly engage them in the learning process. Although we are "both strong proponents of student leadership in promoting academic integrity... [we are convinced that] faculty members have a critical role to play as well” (McCabe and Pavela, 2004, p. 12). 
Indeed, one of the most obvious lessons I have learned from my research is the wisdom of a community-wide approach to promoting academic integrity, with students and faculty exercising the primary responsibility. With this thought in mind, I conclude with some comments about institutional strategies to promote academic integrity.

\section{Institutional Strategies to Promote Student Academic Integrity}

Having attended a university with a traditional academic honor code as an undergraduate, I have always had a bias toward honor code strategies. However, when I first surveyed students in this project in the fall of 1990, I quickly learned that while honor codes may still be an effective strategy, they are not a panacea. See McCabe andTrevino (1993) for a summary of this survey, as well as a detailed definition of traditional honor codes - strategies that generally mandate unproctored exams, the use of some form of pledge that students are asked to sign stating they have not cheated on a particular piece of academic work, a student majority on the hearing board which hears cases of alleged cheating, and, on a declining number of campuses, a requirement or expectation that students will report any peers they may see cheating. While self-reported levels of all forms of cheating were lower on honor code campuses, this research yielded two important results. First, while a campus honor code may have a significant impact on the peer culture, it is the peer culture itself (student perceptions of how faculty and other students feel and behave with regard to academic integrity) that appears to be the most significant factor in influencing the level of academic dishonesty. The second important finding was that strong positive cultures of academic integrity have been achieved on campuses without honor codes and some campuses with honor codes, typically where the tradition but not the reality survives, have above average levels of academic dishonesty.

A number of campuses, especially the University of Maryland at College Park, have responded to such findings about traditional honor codes by developing what have come to be called modified honor codes (see McCabe \& Pavela [2000] for a discussion of modified honor codes). While modified, these codes rest on a strong student role in promoting and sustaining a positive culture of academic integrity, they also acknowledge the reality of how difficult this can be on large campuses. In particular, most of the modified codes being introduced on a growing number of campuses in the United States acknowledge the difficulty of using unproctored exams in a class that might include hundreds of students and the very low probability that students will report the cheating of their peers. So, while maintaining a student judiciary and a strong student role in promoting integrity on campus, most modified codes do not mandate unproctored exams or the use of a pledge. Rather, they often make these choices optional with the instructor. Instructors in large first and second year classes are unlikely to utilize these options while faculty in upperclass courses may feel more comfortable doing so with their typically smaller class sizes and students who are clearly interested in the subject matter, since most are majors in the subject area of the course or a highly related discipline. Students understand this is information they truly need to learn and many may have some prior, or anticipated future relationship with the relevant faculty member - perhaps another course s/he teaches in the department or a research relationship. Even on large campuses, at least some of the anonymity normally associated with large campuses is diminished at the department level. Another difference often found between modified and traditional academic honor codes is the type of sanctions employed. While many traditional codes have strong penalties, modified codes generally place a greater emphasis on penalties which are aimed at rehabilitation rather than punishment. Although strong punishments are available in modified honor codes, and used for multiple or particularly egregious offenses, typically a student will receive some combination of a warning, required attendance at an academic integrity seminar, probation and/or a grade penalty for a first offense under a modified honor code. While campuses with modified codes may abhor cheating as much as those with traditional codes, they see student cheating (at least a first offense) as a teachable moment.

But what traditional and modified honor codes have in common is a community-wide emphasis on the ideal of academic integrity and an acknowledgement of the critical role students can and should play in strategies to reduce cheating. Communicating to students that the institution cares about the issue seems to be an important first step as some, and perhaps many, students have legitimate questions about the role of integrity in today's world. The constant flow of media reports about lapses in integrity among politicians, academics, business people, and just about every other profession one can think of, can create the belief that everyone 
cheats to get ahead and if you want to be competitive and thrive in today's world, you'll have to do the same. Universities may provide our last chance to deliver a different message to young adults, and whether a campus uses an honor code or another approach, the important thing is that each campus does something. The optimal choice will depend heavily on the unique culture and tradition that exists on campus.

Don McCabe is a Professor of Management and Global Business at Rutgers University. He has done extensive research on college cheating, surveying over 100,000 students in the U.S. and Canada. He has also surveyed over 18,000 high school students. His work has been published widely in business, education and sociology journals and he is founding president of the Center for Academic Integrity based at Duke University.

\section{References}

McCabe, D.L., \& Pavela, G. (2000). Some good news about academic integrity. Change, 33(5), 32-38.

McCabe, D.L., \& Pavela, G. (2004). The [updated] principles of academic integrity. Change, 36(3), 10-15.

McCabe, D.L., \& Trevino, L.K. (1993). Academic dishonesty: Honor codes and other contextual influences. Journal of Higher Education, 64(5), 522-538.

McCabe, D.L., \& Trevino, L.K. (1995). Cheating among business students: A challenge for business leaders and educators. The Journal of Management Education, 19(2), 205-218.

McCabe, D.L., \& Trevino, L.K. (1996). What we know about cheating in college: Longitudinal trends and recent developments. Change, 28(1), 28-33.

McCabe, D.L., Trevino, L.K., \& Butterfield, K.D. (2001). Cheating in academic institutions: A decade of research. Ethics \& Behavior, 11(3), 219-232.

McCabe, D.L., Trevino, L.K., \& Butterfield, K.D. (2004). Academic integrity: How widespread are cheating and plagiarism? In D.R. Karp \& T. Allena (Eds.), Restorative justice on the college campus: Promoting student growth, and responsibility, and reawakening the spirit of campus community. Charles C. Thomas, Springfield, IL, 130-141. 